\title{
Increased dispersion of refractoriness in the absence of QT prolongation in patients with mitral valve prolapse and ventricular arrhythmias
}

\author{
Robert G Tieleman, Harry J G M Crijns, Ans C P Wiesfeld, Jan Posma, \\ Hans P M Hamer, Kong I Lie
}

\begin{abstract}
Background-The mechanism responsible for the reported high incidence of ventricular arrhythmias in mitral valve prolapse is not clear. Electrocardiographic studies show an increased occurrence of repolarisation abnormalities on the 12 lead surface electrocardiogram, indicating regional differences in ventricular recovery. The purpose of this study was to investigate whether dispersion of refractoriness was an arrhythmogenic mechanism.
\end{abstract}

Methods-QT dispersion was measured in 32 patients with echocardiographically documented mitral valve prolapse and ventricular arrhythmias on 24 hour Holter recordings. QT dispersion was defined as the difference between the maximum and minimum average $Q T$ interval in any of the 12 leads of the surface electrocardiogram. QT dispersion corrected for heart rate was calculated by Bazett's formula. The results were compared with the data from 32 matched controls without a history of cardiac disease. Patients taking drugs that influence the QT interval and patients with a QRS duration $>120 \mathrm{~ms}$ were excluded.

Results-QT dispersion was greater in patients with mitral valve prolapse than in matched controls (60 (20) $v 39$ (11 ms) respectively, $P \leqslant 0.001)$ as was corrected QT (64 (20 ms) v $43(12 \mathrm{~ms})$ respectively, $P \leqslant 0.001$ ). There was no significant difference in minimum or maximum QT intervals between the two groups.

Conclusions-QT dispersion on the 12 lead surface electrocardiogram was greater in patients with mitral valve prolapse with ventricular arrhythmias than in normal controls, but the maximum QT interval was not increased. The results accord with the hypothesis that regional shortening and lengthening of repolarisation times in patients with mitral valve prolapse may account for the increased dispersion of refractoriness.

(Br Heart f 1995;73:37-40)

Keywords: mitral valve prolapse; ventricular arrhythmia; QT dispersion

Ventricular arrhythmias are common in patients with mitral valve prolapse. ${ }^{1-5}$ This may be related to the increased risk of sudden death in mitral valve prolapse. ${ }^{6-10}$ The ventricular arrhythmias could be caused by triggered activity or dispersion of refractoriness leading to reentry. There are several reports of repolarisation abnormalities such as prolongation of the QT interval corrected for heart rate, ST segment disorders, and inverted $T$ waves, most frequently in the inferolateral leads of the 12 lead surface electrocardiogram. ${ }^{138-14}$ This may indicate regional differences in ventricular electrical recovery, which is widely acknowledged as a substrate for serious ventricular arrhythmias. ${ }^{15} 16$

QT dispersion on the 12 lead surface electrocardiogram may reflect spatial differences in myocardial recovery times. ${ }^{17-19}$ To investigate whether dispersion of refractoriness is an arrhythmogenic mechanism in patients with mitral valve prolapse we measured QT intervals in patients with mitral valve prolapse and ventricular arrhythmias on 24 hour Holter monitoring. QT dispersion and heart rate corrected QT dispersion in patients with symptomatic mitral valve prolapse were compared with dispersion of refractoriness in sex and age matched controls without a history of cardiac disease.

\section{Patients and methods}

\section{PATIENTS}

From the hospital's computerised diagnosis databank, ${ }^{20}$ we retrieved the files of 220 consecutive patients with mitral valve prolapse on echocardiography, who visited our clinic from January 1988 to December 1991. Mitral valve prolapse was defined as a systolic displacement ( $\geqslant 3 \mathrm{~mm}$ ), seen by cross sectional echocardiography, of at least one of the mitral valve leaflets through the plane of the annulus into the left atrium. To avoid oversensitive diagnosis of mitral valve prolapse we studied only patients (123) with an additional midsystolic click or late to pansystolic murmur on auscultation. Forty nine patients did not undergo Holter monitoring. Eighteen patients were excluded because they had ischaemic heart disease. A further 19 patients were excluded because they had atrial flutter or fibrillation $(n=7)$ or a QRS duration $\geqslant 120 \mathrm{~ms}$ $(n=5)$ or because they were taking drugs that influence QT interval (Vaughan Williams class 1 and 3 drugs, $n=9$ ). All these conditions interfere with QT measurements. Five of the remaining 37 patients had no ventricular arrhythmic events on 24 hour Holter monitoring. This left a final study population of 32 patients. 
All 32 patients with mitral valve prolapse had symptoms. Palpitation was the most common indication for Holter monitoring (75\%), followed by atypical chest pain (63\%), presyncope $(34 \%)$ and syncope $(16 \%)$.

The sex and age matched controls were healthy subjects from the surgical day care unit who were examined before general anaesthesia for minor elective surgical procedures. We excluded patients with known disease of the upper abdomen (for example, gallbladder disease, pancreatitis), because these conditions can cause electrocardiographic abnormalities. None of the controls had a history of cardiac disease or arrhythmias, and we excluded controls with ischaemic heart disease, those taking drugs that influence the QT interval, and those with a QRS duration $\geqslant 120$ ms. In total, 32 patients with mitral valve prolapse and 32 controls were selected.

\section{ELECTROCARDIOGRAPHIC MEASUREMENTS}

The electrocardiograms of all patients with mitral valve prolapse and controls were analysed by two independent observers (JP and RT) who were not aware of the clinical condition of the patients. The presence of ventricular arrhythmias or STU abnormalities did not interfere with an unbiased measurement. Differences in reading were resolved by consensus. RR, QRS, and QT intervals were measured by hand. QT intervals were measured from the start of the QRS to the end of the $T$ wave, defined as a return to the T-P baseline. When $U$ waves were present the QT was measured to the nadir of the curve between the $\mathrm{T}$ and $\mathrm{U}$ wave. QT dispersion was determined as described previously. ${ }^{17-19}$ Three consecutive cycles were measured in each of the 12 electrocardiogram leads, and a mean QT per lead was calculated from the three values. QT dispersion was defined as the difference between the maximum and minimum QT occurring in any of the 12 electrocardiogram leads. We calculated heart rate corrected QT dispersion (QTc dispersion) using Bazett's formula (QTc $=\mathrm{QT}$ interval in milliseconds divided by the square root of $R R$ interval in seconds). ${ }^{21}$ When there was no definite end to the $T$ wave or interfering premature complexes the lead concerned was not analysed.

In addition, we studied the correlation

Patient characteristics and electrocardiographic variables (mean (ISD))

\begin{tabular}{lccl}
\hline Variable & MVP & Controls & $P$ value \\
\hline Number & 32 & 32 & \\
Age (y) & $47(16)$ & $47(15)$ & NS \\
Male/female & $16 / 16$ & $16 / 16$ & NS \\
RR (ms) & $862(144)$ & $826(171)$ & NS \\
QRS (ms) & $90(13)$ & $86(7)$ & NS \\
QT min (ms) & $363(29)$ & $373(31)$ & NS \\
QTc min (ms) & $393(31)$ & $413(20)$ & NS \\
QT max (ms) & $423(35)$ & $411(36)$ & NS \\
QTc max (ms) & $458(30)$ & $456(25)$ & NS \\
QT-d (ms) & $60(20)$ & $39(11)$ & $<0.001$ \\
QTc-d (ms) & $64(20)$ & $43(12)$ & $<0.001$ \\
\hline
\end{tabular}

QTc, heart rate corrected QT interval; QT-d, QT dispersion QTmax, maximal average QT on 12 lead surface electrocardiogram; QTmin, minimal average QT on 12 lead surface electrocardiogram. between the number of ventricular (premature) arrhythmias on 24 hour Holter recordings and QTc dispersion in the patients with mitral valve prolapse. QTc dispersion was calculated in patients with palpitation, collapse, presyncope, or chest pain and compared with QTc dispersion in the total group of patients with mitral valve prolapse.

\section{STATISTICAL ANALYSIS}

Data are reported as mean (1 SD). We used the unpaired Student's $t$ test to compare both groups and the Spearman rank-sum test and linear regression analysis for the correlation study. $P$ values $\leqslant 0.05$ were regarded as significant.

\section{Results}

The table shows the characteristics and electrocardiographic variables of both groups. QT dispersion was significantly greater in the patients with mitral valve prolapse than in the matched controls $(P<0.001)$, even after correction for heart rate. Because the mean number of leads analysed per electrocardiogram was similar in both groups no correction was made for the number of leads analysed.

The incidence of ventricular arrhythmias in the patients with mitral valve prolapse on 24 hour Holter monitoring was classified according to Lown. ${ }^{22}$ The median number of premature ventricular complexes was 142/24 hour (range 1-26, 544). Twenty one patients had fewer than 30 premature ventricular complexes/24 hour and 11 had more than 30 premature ventricular complexes/hour. Sixteen patients had couplets of premature ventricular complexes. Non-sustained ventricular tachycardia (heart rate $>100$ beats per minute lasting $<30$ seconds) was present in 11 patients. There were no patients with sustained ventricular tachycardia or fibrillation.

There was no significant relation between the number of premature ventricular complexes during 24 hour Holter monitoring and the QTc dispersion in patients with mitral valve prolapse. QTc dispersion in patients with couplets of premature ventricular complexes or non-sustained ventricular tachycardia resembled QTc dispersion in patients with only isolated premature ventricular complexes. The median QTc dispersion in patients with couplets of premature ventricular complexes was $55 \mathrm{~ms}$ (range 27-110 ms) and $56 \mathrm{~ms}$ (range $27-83 \mathrm{~ms}$ ) in patients with non-sustained ventricular tachycardias. Finally, QTc dispersion in patients with symptoms was comparable to the average QTc dispersion in all patients with mitral valve prolapse, but much higher than QTc dispersion in matched controls without these symptoms.

\section{Discussion}

This study shows that QT and QTc dispersion were higher in patients with mitral valve prolapse and ventricular arrhythmia than in a similar group of controls. In addition, the 
present report is the first to suggest that this dispersion is the consequence of the combination of short minimum and long maximum QT intervals rather than exclusive QT prolongation. Earlier studies indicated that QTc dispersion reflects underlying regional differences in the recovery of ventricular excitability. ${ }^{17-19} 23$ Recently, an association has been reported between ventricular arrhythmias and QTc dispersion. ${ }^{24}$ Therefore the increased dispersion of refractoriness could well be the arrhythmogenic mechanism in mitral valve prolapse. However, the present study did not detect a direct correlation between the increased dispersion of refractoriness in mitral valve prolapse and the number of spontaneously occurring ventricular arrhythmias or the incidence of complaints such as palpitation, presyncope, or syncope. This could be due to the retrospective design of the study.

There are several possible explanations for increased differences in myocardial recovery times in patients with mitral valve prolapse. One explanation relates to autonomic dysfunction, which is present in most patients with mitral valve prolapse. ${ }^{25}$ Several studies showed high circulating catecholamines, increased responsiveness to catecholamines, abnormal catecholamine regulation and baroreflex modulation, and activation of atrial natriuretic peptide in patients with mitral valve prolapse. Excitation of the sympathetic nervous system increased dispersion of refractoriness in an animal model both through regional shortening and regional prolongation of ventricular refractoriness. ${ }^{26}$ Furthermore, there is anecdotal evidence that $\beta$ adrenergic blockade suppresses symptoms of mitral valve prolapse. ${ }^{27}$

Another cause of increased dispersion of refractoriness may be regional differences in wall stress. In patients with mitral valve prolapse traction on the chordae tendineae may be higher because of the increased valve surface area and this could induce regional differences in wall stress. Experimental evidence from studies in animals and humans showed shortening of the action potential or spontaneous afterdepolarisations when myocardial wall stress was increased. ${ }^{28}$ This phenomenon, called mechanoelectric feedback or contraction-excitation feedback is thought to be an arrhythmogenic mechanism in patients with heart failure..$^{29}$ It will increase the inhomogeneity of electrical recovery when deteriorating mechanical dysfunction increases wall stress. The regional differences in myocardial wall stress in mitral valve prolapse may have a similar effect.

A third possibility is that generation of delayed afterdepolarisations and triggered activity in the basal parts of the altered mitral valve leaflets itself increases QTc dispersion, as suggested by Shenoy et $a l^{30}$ and as demonstrated in vitro by Wit et al. ${ }^{31}$

The hypothesis that mitral valve prolapse is an atypical form of the adrenergic dependent long QT syndrome is based on observations of prominent $U$ waves, QTc prolongation, and typical ventricular arrhythmias (torsades de pointes) in mitral valve prolapse. ${ }^{32}$ The occurrence of adrenergic dependent ventricular arrhythmias also suggests that delayed afterdepolarisations play a part in arrhythmogenesis in mitral valve prolapse. In theory, afterdepolarisations could well be the arrhythmogenic factor in mitral valve prolapse. However, they would cause regional QT prolongation, and although in some earlier electrocardiographic studies the incidence of QTc prolongation in mitral valve prolapse was high, ${ }^{14913}$ we could not demonstrate an increased incidence of prolonged QTc in patients with mitral valve prolapse compared with controls. This accords with other controlled studies. ${ }^{1233}$ Earlier studies ${ }^{14913}$ did not include normal controls. Despite a normal incidence in QTc prolongation, however, we found an increased QTc dispersion, which could serve as an arrhythmogenic mechanism too.

In this study we measured QT intervals in all leads and found that QTc dispersion was increased in patients with ventricular arrhythmias and mitral valve prolapse. Surface electrocardiographic QT measurement may be subject to errors, but variation in interlead measurements far outweighs inaccuracies introduced by any other factor including interobserver variation. ${ }^{23}$ Our study indicates that increased QT dispersion may be due to regional shortening combined with lengthening of repolarisation times. As outlined above, this shortening of action potential duration may be caused by increased adrenergic stimulation or locally increased wall stress. Other evidence, however, suggests that induction of afterdepolarisations prolongs action potential duration. Therefore, the mechanism of ventricular arrhythmias may vary in different patients with mitral valve prolapse. The fact that $\beta$ blockers can improve symptoms of mitral valve prolapse supports both of the proposed arrhythmogenic mechanisms. $\beta$ Blockers prevent the induction of delayed afterdepolarisations in patients with the adrenergic dependent long QT syndrome and also reduce the dispersion of refractoriness. Furthermore, because in our study QTc dispersion was commonly increased in the absence of significant QTc prolongation, there is a new theoretical basis for examining agents, such as class III antiarrhythmic drugs, that reduce dispersion of refractoriness in patients with mitral valve prolapse.

1 Gooch AS, Vincencio F, Maranhao V, Goldberg H. Arrhythmias and left ventricular asynergy in the prolaps-
ing mitral leaflet syndrome. Am $₹$ Cardiol 1972;29:
$611-20$.

So, Fitzgerald JW, Goodman DJ, Schroeder JS, Harrison DC. Arrhythmias in patient

3 Campbell RWF, Godman MG, Fiddler GI, Marquis RM, Julian DG. Ventricular arrhythmias in syndrome of Julian DG. Ventricular arrhythmias in syndrome of
balloon deformity of mitral valve. Br Heart $\mathcal{f} 1976 ; 38$ : balloon

4 Wei JY, Bulkley BH, Shaeffer AH. Mitral-valve prolapse syndrome and recurrent ventricular tachyarrhythmias: a syndrome and recurrent ventricular tachyarrhythmias: a malignant variant refractory to co
apy. Ann Intern Med 1978;89:6-9.

5 Savage DD, Levy D, Garrison RJ, Castelli WP, Kligfield P, Devereux RB, et al. Mitral valve prolapse in the general P, Devereux RB, et al. Mitral valve prolapse in the general
population. 3. Dysrhythmias. Am Heart $\mathcal{f} 1983 ; 106$ : population. 
6 Kligfield P, Levy R, Devereux RB, Savage DD. Arrhythmias and sudden death in mitral valve prolapse. Am Heart $\mathcal{F}$ 1987;113:1298-307.

7 Nishimura RA, McGoon MD, Shub C, Miller FA, Ilstrup DM, Tajik AJ. Echocardiographically documented mitral valve prolapse. $N$ Engl $₹$ Med $1985 ; 313: 1305-9$.

8 Winkle RA, Lopes MG, Popp RL, Hancock EW. Lifethreatening arrhythmias in the mitral valve prolapse syndrome. Am $\mathcal{F}$ Med 1976;60:961-7.

9 Jeresaty RM. Sudden death in the mitral valve prolapseclick syndrome. Am $\mathcal{F}$ Cardiol 1976;37:317-18.

10 Pocock WA, Bosman CK, Chesler E, Barlow JB, Edward $J E$. Sudden death in primary mitral valve prolapse. $A m$ Heart $\mathcal{f} 1984 ; 102: 378-82$.

11 Lobstein HP, Lawrence DH, Curry GC, Mullins CB Electrocardiographic abnormalities and coronary arteriograms in the mitral click-murmur syndrome. N Engl F Med 1973;289:127-31.

12 Bhutto RZ, Barron JT, Liebson PR, Uretz EF, Parillo JE. Electrocardiographic abnormalities in mitral valve prolapse. Am $千$ Cardiol 1992;70:265-6.

13 Gardin JM, Isner JM, Ronan JA, Fox SM. Pseudoischemic "false positive" S-T segment changes induced by hyperventilation in patients with mitral valve prolapse. $A m \mathcal{F}$ ventilation in patients

14 Meyers DG, Vallone NL, Engel TR. Repolarization abnormalities in mitral valve prolapse. Am Heart $\mathcal{f}$

15 Han J, Moe GK. Non-uniform recovery of excitability in ventricular muscle. Circ Res 1964;14:44-60.

16 Kuo CS, Munakata K, Reddy CP, Surawicz B. Characteristics and possible mechanism of ventricular arrhythmia dependent on the dispersion of action potential durations. Circulation 1983;67:1356-67.

17 Day CP, McComb JM, Campbell RWF. QT dispersion: an indication of arrhythmia risk in patients with long QT intervals. Br Heart $₹$ 1990;63:342-4.

18 Day CP, McComb JM, Matthews J, Campbell RWF. Reduction in QT dispersion by sotalol following myocardial infarction. Eur Heart f 1991;12:423-7.

19 Day CP, McComb JM, Campbell RWF. QT dispersion in sinus beats and ventricular extrasystoles in normal hearts. Br Heart $\mathcal{F}$ 1992;67:39-41.

20 Dijk WA, Haagen FDM. INFOCOP, Information system for catheterised and operated patients. Computers in Cardiology, Long Beach California: IEEE Computer
Society, 1984:241-4.

21 Bazett HC. An analysis of the time-relations of electrocardiograms. Heart 1920;7:353-70.

22 Lown B, Wolf M. Approaches to sudden death from coronary heart disease. Circulation 1971;44:165-70.

23 Cowan JC, Yusoff $\mathrm{K}$, Moore M, Amos PA, Gold AE, Bourke JP, et al. Importance of lead selection in QT interval measurement. Am $₹$ Cardiol 1988;61:83-7.

24 Hii JTY, Wyse DG, Gillis AM, Duff HJ, Solylo MA, Mitchell LB. Precordial QT interval dispersion as a marker of torsade de pointes. Disparate effects of class la antiarrhythmic drugs and amiodarone. Circulation 1992;86:1376-82.

25 Boudoulas $\mathrm{H}$, Wooley CF. Hyperadrenergic state, autonomic dysfunction, and pathogenesis of symptoms in mitral valve prolapse syndrome. In: Boudoulas $\mathbf{H}$, Wooley CF, eds. Mitral valve prolapse and the mitral valve prolapse syndrome. Mount Kisco, NY: Futura Publishing, 1988:479-510.

26 OptHof T, Ramdat Misier AR, Vermeulen JT, Frank RGJ, van Capelle FLJ, Coronol R, et al. Dispersion of "refractoriness" in canine ventricular myocardium: Effects of sympathetic stimulation. Circ Res 1991;68:1204-15.

27 Alpert MA. Mitral valve prolapse. $B r$ Med $¥$ 1993;306:

28 Lab MJ. Contraction-excitation feedback in myocardium. Physiological basis and clinical relevance. Circ Res 1982; 50:757-66.

29 Dean JW, Lab MJ. Arrhythmia in heart failure: role of mechanically induced changes in electrophysiology. Lancet 1989;i:1309-12.

30 Shenoy MM, Hariman RJ, Balla S, Punatar HK. Triggered activity as a possible mechanism of ventricular tachycardia in mitral valve prolapse. Am Heart 7 1986; 112:1339-42.

31 Wit AL, Fenoglio J, Hordof AJ, Reetsma K. Ultrastructure and transmembrane potentials of cardiac muscle in the human anterior mitral valve leaflet. Circulation 1979;59:1284-92.

32 Jackmann WM, Friday KJ, Anderson IL, Aliot EM, Clark $M$, Lazzara $R$. The long QT syndromes: a critical review, new clinical observations and a unifying hyporeview, new clinical observations and a unifyis

33 Cowan MD, Fye WB. Prevalence of QTc prolongation in women with mitral valve prolapse. Am f Cardiol 1989; 63:133-4. 